\title{
Parametrized $\bar{\partial}$ Operator on Pseudoconvex Sets $\left({ }^{*}\right)(* *)$.
}

\author{
Claudio Rea (L'Aquila)
}

\begin{abstract}
Sunto. - Si dimostra l'esistenza della soluzione per l'operatore di Oaushy-Riomann parametrizzato, nel caso continuo per varietà fortemente pseudoconvesse e nel caso differenziabile per varietà di Stein.
\end{abstract}

\section{\$1. - Introduction.}

For a given smoothly parametrized operator $P_{t}$ of constant degree there is generally no continuously parametrized solution of the equation $P_{t} u_{t}=f_{t} \in R P_{t}$, even when $f_{t}$ is regular with respect to the pair parameter-variable. A simple example is given by the equation $u_{\theta}+t^{2} \varrho u_{\varrho} \equiv\left(t^{2} x-y\right) u_{x}+\left(t^{2} y+x\right) u_{y}=t$ on the plane. In the present paper we show that the above fact does not happen if $P_{0}$ is the $\vec{\partial}$ operator on any strongly pseudoconvex set [prop. (4.1)] and we give a smoothly parametrized solution for the Stein case $\left.{ }^{(1}\right)$. We work on a $O^{\infty}$ family $W$ of complex manifolds $X_{t}$; the space of the parameter $t$ is a ball $B$ of $\mathrm{R}^{m}$ and could be reduced to a segment without any real loos of generality. Consider the equation

$$
\bar{\partial} v=f
$$

(it should be written $\bar{\partial}_{t} v_{t}=f_{t}$ ) on forms with values in a $C^{\text {co }}$ vector bundle $E$ such that $E_{t}=E \mid X_{t}$ is a holomorphic bundle (precise details about the algorithm are given in next section). In section 6 we shall prove the following

(1.2) THeoRem. - Let $\mathfrak{W}=\left\{X_{t}\right\}_{t \in B}$ be a $C^{\infty}$ family of complex manifolds with $X_{0}$ Stein. Each compact set $K \subset X_{0}$ has a neighborhood $\Omega$ in $W$ such that, for every smooth family $\left(f_{t}\right)_{t \in B}$ of $C^{\infty}(p, q+1)$-forms with values in the fiber bundle $E_{t}$ which satisfies $\bar{\partial}_{t} f_{t}=0$, there exists on $\Omega$ a $C^{\infty}$ family $\left(v_{t}\right)_{t \in B}$ of $(p, q)$-forms which resolves (1.1).

Equation (1.1) is considered here with vector bundle valued forms out of necessity and not for excessive, apparent generality. Actually our proof goes through the existence of a continuous solution for the holomorphic tangent bundle which is, by the way, just of the type of $E$.

(*) Entrata in Redazione il 20 maggio 1975.

(*) Supported by C.N.R. research groups.

(1) Indeed our manifold at time 0 need not to be Stein but only "limit of Stein * (i.e. every compact set has a Stein neighborhood), but since we do not know wether this hypothesis is really more general, we assume it to be Stein. 
In section 7 we give two applications of this theorem:

pseudorigidity of Stein manifolds is proved for $C^{\infty}$ deformations,

two proofs of the complex Frobenius theorem of L. Nirenberg are given.

The first one is based on pseudorigidity and Newlander-Nirenberg theorem; the second one (only sketched) is a direct application of the present methods to KoHN proof ([7]) of the integrability theorem for almost complex structures.

\$2. - a) Our total space is the product of a connected differential manifold $X$ and an open ball $B$ of $\mathbb{R}^{m}$. At each point $(x, t)$ of $X \times B$ is defined a tensor $J: T_{x} X \rightarrow T_{x} X$ with $J^{2}=-i d$ which depends smoothly on $(x, t)$ and satisfies the integrability conditions which are necessary (and sufficients) to give each $X \times\{t\}$ the structure of a complex manifold. We will note this complex manifold by $X_{t}$ and the product $X \times B$ whith this additional structure by $w$. $W$ is a $C^{\infty}$ family of complex manifolds. It is useful to introduce the trivial map $\mathscr{G}: X \times B \rightarrow W$ and the natural projection $\pi: \mathfrak{W} \rightarrow B$. By the Frobenius-Nirenberg theorem [8] the structure of family of complex manifolds given by the tensor $J$ can be described by an atlas $\left(W_{j}, \chi_{j}\right)$ of diffeomorphisms

$$
\chi_{j}: W_{j} \rightarrow \text { open subset of } \mathbb{C}_{z_{j}}^{n} \times \mathbb{R}_{t}^{m}
$$

in such a way that the $t$-component of $\chi_{j}(x, t)$ is $t$, the coordinates $z_{h}$ are holomorphic functions of $z_{j}$ in $W_{j} \cap W_{h}$ for fixed $t$ and the complex structure on $X \times\{t\}$ is just the same that was induced by $J$, i.e. $X_{l}$.

Let $\varphi_{0} \in C^{\infty}\left(X_{0}\right)$ be the function, plurisubharmonic at $\left\{\varphi_{0}=0\right\}$, defining $\Omega_{0}=\left\{\varphi_{0}<0\right\}$ and $\varphi$ be its trivial extension to $w$ given by $\varphi[\mathcal{G}(x, t)]=\varphi_{0}(x)$. The restriction $\varphi_{t}$ of $\varphi$ to $X_{t}$ defines a set $\Omega_{t} \subset X_{t}$ which is obviously still strongly pseudoconvex for $|t|$ small enough, say $|t|<\delta$. Thus we can suppose, up to an unessential restriction, that the basis ball $B$ is just $|t|<\delta$. The open subset $\Omega$ of $w$ given by $\varphi<0$ is the neighborhood of $\Omega_{0}$ mentioned in theorem (1.2).

If $\Omega_{0}$ is Stein, a classical procedure shows that, up to a convenient restriction of $B$, by taking a sufficiently increasing and convex function $F: \mathbb{R} \rightarrow \mathbb{R}, \psi=F(\varphi)$ and the minimum eigenvalue $\lambda_{\psi}$ of its Levi form can be assumed to be greater than any given continuous function on $\Omega$, [9].

b) The complexified tangent bundle of $w$ has a sub-bundle $\boldsymbol{T}$ of vectors which are linear combinations of $\partial / \partial z_{1}, \ldots, \partial / \partial z_{n}$. The restriction $\boldsymbol{T}_{t}$ of $\boldsymbol{T}$ to $X_{t}$ is the usual complex tangent bundle. Let $A^{10}$ be the dual bundle of $T$ and $\Lambda^{01}$ its conjugate bundle. At each point $\zeta \in W$, the fibers $\Lambda^{10}(\zeta)$ and $\Lambda^{01}(\zeta)$ generate an exterior algebra $\oplus_{1}^{n} \Lambda^{p \alpha}(\zeta)$. The vector space $\Lambda^{p \alpha}(\zeta)$ is the fibre of a bundle $\Lambda^{p a}$ over $w$ whose restriction $\Lambda_{t}^{p a}$ to $X_{t}$ is the usual bundle of $(p, q)$-forms on $X_{t}$. Note that $\Lambda^{p a}=\Lambda^{p 0} \otimes \Lambda^{0 q}$. Let $E$ be a complex $C^{\infty}$ vector bundle on $W$ whose restriction $E_{t}$ to $X_{t}$ is a holo- 
morphic bundle. Two examples of such bundles are $\boldsymbol{T}$ and $\Lambda^{p 0}$. In this paper we shall consider $(p, q)$-forms with values in an arbitrary bundle like $E$ above, i.e. with cross sections of $A^{p 0} \otimes E$. Since $A^{p 0} \otimes E$ is still of the same type of $E$, it is no restriction to assume $p=0$. For any subset $A$ of $w$, let $C_{a}^{k}(A)$ be the space of $O^{k}$ sections of $A^{0 \alpha} \otimes E O^{\infty}$ by respect to the z's. Over any open neighborhood of $A$, $0 \leqslant k \leqslant \infty$. Then the operator $\bar{\partial}: C_{a}^{k}(W) \rightarrow C_{a+1}^{k}(\mathfrak{W})$ is well defined and its restriction $\bar{\partial}_{t}$ to $X_{t}$ is the usual $\bar{\partial}$ operator on $X_{t}$ for $(0, q)$-forms with values in $E_{t}$. Thus the form $f$ appearing in (1.1) belongs to $O_{a+1}(W) \equiv C_{q+1}^{\infty}(W)$ and $u$ belongs to $O_{q}(\Omega)$. $\equiv C_{a}^{\infty}(w)$.

\$ 3. - We recall briefly some classical results which can be found in [7] and [4], concerning the Laplace-Beltrami operator for a smooth, strongly pseudoconvex open subset $\Delta$ of a hermitian manifold $X$. Let be $\Delta \equiv\{\varphi<0\}$, where $\varphi$ is a smooth function on $X ; d \varphi$ does not vanish at the boundary $\partial \Delta \equiv\{\varphi=0\}$. We shall consider $(0, q)$-forms on $X$ with values in a holomorphic hermitian vector bundle $E$; the space of those among them which are $C^{\infty}$ will be noted by $O_{\alpha} . D_{a}$ will denote the subspace of those with compact support. The hermitian product $\langle\cdot, \cdot\rangle$ on the fiber $E_{x} \otimes A_{x}^{0 u}$ is defined by putting $\langle v \otimes \chi, u \otimes \theta\rangle=\langle v, u\rangle\langle\chi, \theta\rangle,(\cdot, \cdot)=\int_{\boldsymbol{\Delta}}\langle\cdot, \cdot\rangle \cdot$ $\cdot e^{-\varphi} d V$ is the $\mathfrak{L}^{2}$ hermitian product, $\|u\|=(u, u)^{\frac{1}{4}}$ is the $\mathfrak{L}^{2}$ norm. Let be $T$ and $\$$ the closed $\mathfrak{L}^{2}$ extension of the operators $\bar{\partial}: C_{q}(\bar{A}) \rightarrow C_{q+1}(\bar{A})$ and $\bar{\partial}: O_{q+1}(\bar{A}) \rightarrow C_{q+2}(\bar{A})$. The main result of the theory says that the strong pseudoconvexity of $\Delta$ implies that the operators $S, T^{*}$ and $L=T T^{*}+S^{*} S$ have a closed range ([7]), hence we have

$$
\|u\| \leqslant C\|L u\|, \quad \text { for } u \in D_{L}
$$

$D_{L}$ is the domain of $L$.

REMARK. - The constant $C$ is a continuous function of the metrics on $\boldsymbol{T}$ and $E$, of $\varphi$ and their derivatives.

We fix now orthonormal frames $\omega^{\alpha}(x)$ and $e_{a}(x)$ for $\boldsymbol{T}_{x}^{*}$ and $E_{x}$, depending smoothly on $x$ in each coordinate neighborhood. Blocks of indices are always supposed to be strictily increasing, $\omega^{A}$ stands for $\omega^{\alpha_{1}} \wedge \ldots \wedge \omega^{\alpha_{p}}$, with $A=\alpha_{1}, \ldots, \alpha_{p},|A|=p$, and we put $\bar{\omega}_{a s}^{A}=e_{a} \otimes \bar{\omega}^{A}$. Thus the punctual hermitian product between the vector valued forms $f=\sum_{a A} f_{A}^{a} \omega_{a}^{A}$ and $g=\sum_{a A} g_{A}^{a} \omega_{a}^{A}$ is given by $\langle f, g\rangle=\sum_{a A} f_{A}^{a} g_{A}^{a}$. The operator $\nabla_{\alpha}$ on functions is defined by $\bar{\partial} u=\sum \bar{\nabla}_{\alpha} u \cdot \bar{\omega}^{\alpha}$. By putting $\delta_{\alpha} u=e^{\varphi} \nabla_{\alpha}\left(u e^{-\varphi}\right)$ we get the formal adjoint $\vartheta$ of $\bar{\partial}$ on $\mathcal{D}_{q}(X) . \vartheta$ is defined by $(g, \bar{\partial} f)=(\vartheta g, f)(f$ and $g$ are smooth and one of them has compact support), and explicitely given by $\vartheta g=-\sum_{\alpha a A} \delta_{\alpha} g_{\alpha A}^{a} \omega_{a}^{A}+$ + linear part. The Green formula is

$$
(f, \bar{\partial} g)=(\vartheta f, g)+\int_{\partial \Delta}\langle f, g \wedge \bar{\partial} \varphi\rangle d S, \quad \text { with } f \in O_{a+1}(\bar{\Delta}), g \in C_{q}(\bar{\Delta}),
$$


where $d S$ is a suitable positive measure on $\partial \Delta$. The restriction of $L$ to $\mathfrak{D}_{q}$ is the Laplace-Beltrami operator $\square=\bar{\partial} \vartheta+\vartheta \bar{\partial}$. By successive applications of Green formula (3.3) we get

$$
(f, \square \varphi w)=(\square f, \varphi w)+\int_{\partial \Delta}|\vec{\partial} \varphi|^{2}\langle f, w\rangle d S, \quad \text { for } f, w O_{q}(\bar{\Delta}) .
$$

Proposttion. - The form $f \in C_{Q}(\bar{A})$ is in the domain $D_{L}$ of $L$ if and only if $f=\varphi^{2} g$, with $g \in O_{q}(\bar{d})$.

Proof. $-f$ is in $D_{L}$ if and only if there exists a constant $e$ such that

$$
|(f, \square u)| \leqslant c\|u\|, \quad \text { for each } u \in C_{q}(\bar{\Delta}) .
$$

Take any form $w \in C_{q}(\bar{\Delta})$ and replace $u$ in (3.6) by $\varphi w$. Since we may have $\|q w\| \leqslant 1$, with $w$ arbitrarely large at the boundary, we conclude from (3.4) that $f$ must vanish at $\partial \Delta$ i.e. $f=\varphi h$. Now we conjugate (3.4) with $w$ replaced by $h$ and $f$ by $u$ and we have

$$
(f, \square u)=(\square f, u)-\int_{\partial \Delta}|\bar{\partial} \varphi|^{2}\langle h, u\rangle d S .
$$

The same argument as before says that $h$ must vanish at the boundary i.e. $h=\varphi g$, which means $f=\varphi^{2} g$. Conversely (3.7) shows that, when $f=\varphi^{2} g$, then $|(f, \square u)| \leqslant$ $\leqslant\|\square f\|\|u\| . \quad$ Q.E.D.

Our purpose is to prove the existence of a solution for the perturbed operator $T_{t}$ which is regular with respect to the parameter. Since the parameter regularity is generally easier to prove when the solution is unique, it is more convenient to reduce the problem to one which has the unicity property. The above argument is the only reason we have to employ the Laplace-Beltrami operator $\square$.

All that we need of this theory can be resumed in the following.

(3.8) Propostrion. - If $u$ is the (unique) solution in $D_{L}$ of the equation $L u=f$, with $f \in C_{q}(\bar{A})$ and $\bar{\partial} f=0$, then $u$ is smooth because of the ellipiticity of $L$, and $v=\vartheta u$ is the unique solution of the equation $\bar{\partial} v=f$ which is orthogonal to $\operatorname{ker} T$.

Proof. - It is enough to show that $f=\bar{\partial} \vartheta u$, i.e. that $w=\vartheta \bar{\partial} u$ vanishes. The ranges of $T$ and $T^{*}$ are closed, this implies ([4], p. 140) the inequality

$$
\|g\| \leqslant \text { const }\|T g\|, \quad \text { for each } g \in D_{T} \cap R_{T^{*}} \text {. }
$$

Now the form $w \in C_{q}(\bar{A})$ belongs to $D_{T} \supset C_{q}(\bar{A})$. Moreover, since $u$ vanishes at the boundary $\partial A$ by Prop. (3.5), formula (3.3) gives that $\bar{\partial} u$ is in $D_{T^{*}}$. Thus $w \in R_{T^{*}}$. Finally the condition $\bar{\partial}(f=w+\bar{\partial} \vartheta u)=0$ gives $T w=\bar{\partial} w=0$ and by (3.9) we have $w=0$. Q.E.D. 


\section{\$ 4. - The continuous solution for the tangent bundle $T$.}

We refer to the notation of $\S 2$; consider again the open set $\Omega \subset \mathcal{W}$ of $\S 2 a$ and suppose that $E$ is the bundle $T$ introduced in $\$ 2 b$. A cross section $f \in C_{a+1}(w)$ is given; for every $t \in B$ consider the extended Laplace-Beltrami operator $L_{t}$ relative to the pseudoconvex subset $\Omega_{t}$ of $X_{t}$ as in $\S 3$ and to the function $\varphi_{t}=\varphi \mid X_{t}$. Suppose that $f_{t} \in R L_{t}$, for each $t$. Then as mentioned in (3.8), there exists a unique solution $u_{t} \in D_{t_{t}}$ of the equation $\square u_{t}=f_{t}$. The collection $\left(u_{t}\right)_{t \in B}$ gives obviously a cross section $u$ over $\bar{Q}$ of the bundle $\Lambda^{0 \alpha} \otimes \boldsymbol{T}$. The continuity of $u$ is implicitely proved in [7], p. 144. We give here a more detailed proof wich make use of some inequalities which will be useful later.

$$
\text { Proposition. - } u \text { is a continuous section of } \Lambda^{00} \otimes \boldsymbol{T} \text {. }
$$

Before starting with the proof we need to adapt to our case some classical inequalities for elliptic operators. For every subset $A_{0} \subset \subset X_{0}$, put $A=\mathcal{C}^{-1}\left(A_{0} \times B\right)$ and $A_{t}=A \cap X_{t}=\mathscr{C}^{-1}\left(A_{0} \times\{t\}\right)$. Since $\bar{\Omega}$ is compact it can be covered by a finite number $W_{1}, \ldots, W_{N}$ of cards of the atlas mentioned in $\S 2 a$; in what follows the sets $W_{j}$ will be fixed. A straightforward compactness argument shows that the ray $\delta$ of $B$ can be chosen so small that $\pi W_{j}=B$ for each $j \leqslant N$. We denote by $\tilde{C}_{q}(\bar{A})$ the space of the families $\left\{f_{j}\right\}_{j \leqslant N}$ of $C^{\infty}$ cross sections of $\Lambda^{0 a} \otimes T$ over $W_{j}$ and by $\tilde{C}_{q}\left(\bar{A}_{t}\right)$ the similarly defined space for $t \in B$. Although the elements of those spaces are not sections of $\Lambda^{0 q} \otimes T$, nevertheless the Laplace-Beltrami operator $\square_{t}$ of $X_{t}$ operates on $\tilde{C}_{q}\left(\bar{A}_{\tau}\right)$ and even on $\tilde{C}_{q}\left(\bar{A}_{t}\right)$ with $\tau \neq t$ as we shall see now. In the coordinate neighborhood $W_{j}$ write the operator $\square$ in the form

$$
(\square v)_{A}^{a}(z, t)=\sum_{b B \alpha} L_{A B \alpha}^{a b}(\alpha, t)\left(D^{\alpha} v_{B}^{b}\right)_{\left(z_{2} t\right)} ; \quad b \leqslant N,|B|=q,|\alpha| \leqslant 2,
$$

where $L_{A B \alpha}^{a b}$ are $C^{\infty}$ functions on $\vec{W}_{j}$ and, for each $v_{\tau} \in \tilde{C}_{\alpha}\left(\bar{A}_{\tau}\right)$, put

$$
\left(\square_{t} v_{\tau}\right)_{A}^{a}(z, t)=\sum_{b B \alpha} L_{A B \alpha}^{a b}(z, t)\left(D_{\alpha} v_{B}^{b}\right)_{(z, \tau)} ; \quad b \leqslant n,|B|=q,|\alpha| \leqslant 2
$$

We shall use in $\tilde{C}_{a}\left(\bar{\Omega}_{t}\right)$ and $\tilde{O}_{a}(\bar{A})$ the Sobolev norms given by

$$
\left(\left\|v_{t}\right\|_{s}^{A}\right)^{2}=\left\{\sup (-2 i)^{n} \int_{\bar{A} \cap W_{1}}\left|D^{\alpha} u_{B}^{b}\right|_{(z, t)}^{2} d z \wedge d \bar{z} ;|\alpha| \leqslant s, j \leqslant N, b \leqslant n,|B|=q\right\},
$$

where the derivatives $D^{\alpha}$ are made only with respect to the variables $z, \bar{z}$, and by

$$
\|v\|_{s}^{A}=\sup _{i \in \bar{B}}\left\|v_{i}\right\|_{A}^{s}, \quad\left(v_{i}=v \mid X_{i}\right)
$$


It will be also useful to introduce the position

$$
\left(\left\|v_{\tau}-v_{i}\right\|_{s}^{A}\right)^{2}=\sup \left\{(-2 i)^{n} \int_{A \cap W_{j}}\left[\left.D^{\alpha}\left[v_{B}^{b}(z, \tau)-v_{B}^{b}(z, t)\right]\right|^{2} d z \wedge d \bar{z} ;|\alpha| \leqslant s, j \leqslant N, b \leqslant n,|B|=q\right\} .\right.
$$

From the regularity of the coefficients $L_{A B x}^{a b}$ appearing in (4.2) we get immediatly the following inequalities

$$
\begin{aligned}
& \left\|\left(\square_{\tau}-\square_{t}\right) v_{\tau}\right\|_{s}^{A} \leqslant k_{1}\left\|v_{\tau}\right\|_{s+2}^{A}|t-\tau|, \\
& \left\|\square_{\tau} v_{\tau}\right\|_{s}^{A} \leqslant k_{2}\left\|v_{\tau}\right\|_{s+2}^{A}
\end{aligned}
$$

which are valid for every $t$ and $\tau$ in $B, v \in \tilde{C}_{q}\left(\bar{A}_{\tau}\right)$ and the constants $k_{1}, k_{2}$ do not depend on $t$ and $\tau$.

(4.5) Proposimion. - If $u$ is the above oross section of $\Lambda^{0 a} \otimes T$ then, for every $t \in B$, $\lim _{\tau \rightarrow t}\left\|u_{t}-u_{x}\right\|^{\Omega}=0$.

Proof. - The fiber preserving diffeomorphism $\mathscr{C}: \bar{\Omega} \rightarrow \bar{\Omega}_{0} \times B$ introduced in section $\$ 2 a$ induces a diffeomorphism $\mathscr{G}_{t z}: \bar{\Omega}_{t} \rightarrow \bar{\Omega}_{\tau}$ which is the $\mathcal{C}^{-1}$-image of the trivial map $\bar{\Omega}_{0} \times\{t\} \rightarrow \Omega_{0} \times\{\tau\}$. $\mathcal{G}_{i \tau} \zeta$ depends smoothly on $(\zeta, t, \tau)$. Let $t \in B$ be fixed and let $\mathfrak{G}_{i t}^{a}$ be the composed map

$$
\tilde{G}_{l \tau}^{\alpha}: \Lambda^{0 \alpha} \otimes \boldsymbol{T}_{t} \rightarrow \underset{r+\oplus_{m=q}}{\oplus}\left[\Lambda_{\tau}^{r m} \otimes\left(\boldsymbol{T}_{\boldsymbol{\tau}} \oplus \overline{\boldsymbol{T}}_{\tau}\right)\right] \rightarrow \Lambda^{0 \alpha} \otimes \boldsymbol{T}
$$

The first arrow above is induced by $\mathcal{C}_{t r}$ and the second is the natural projection. The map $\mathscr{C}_{t \tau}^{\alpha}$ induces a map $C_{q}\left(\bar{\Omega}_{t}\right) \rightarrow C_{\alpha}\left(\bar{\Omega}_{\tau}\right)$, is the identity for $t=\tau$, and depends smoothly on all its arguments i.e.: for each $w_{t} \in C_{a}\left(\bar{\Omega}_{t}\right)$ the cross section $w$ of $\Lambda^{0 a} \otimes \boldsymbol{T}$ on $\bar{\Omega}$ given by $w_{\tau}=\sigma_{t \tau}^{a} w_{i}\left(\tau\right.$ varying in $B$ ) is a $C^{\infty}$ extension of $w_{t}$. Since $u_{t} \in D_{L_{t}} \cap C_{q}\left(\bar{\Omega}_{t}\right)$ by prop. (3.5) it is $u_{t}=\phi^{2} w_{t}, w_{t} \in O_{q}\left(\bar{\Omega}_{t}\right)$. The above procedure gives a $C^{\infty}$ extension $w$ of $w_{t}$. Since $w$ is regular up to the boundary of $\Omega$, there exists a constant $k_{3}$ such that

$$
\left\|u_{t}-\varphi^{2} w_{\tau}\right\|_{2}^{\bar{B}} \leqslant k_{3}|t-\tau|, \quad \text { for each } \tau \in B
$$

The choice of $w$ in $O_{q}(\bar{\Omega})$ depends only on $t$ which is fixed here. Thus we have

$$
\left\|\varphi^{2} w_{\tau}\right\|_{2}^{\Omega} \leqslant k, \quad \text { for each } \tau \in B
$$

From formula (3.1) and taking account of the remark (3.2) there exists a constant $C$ such that we have

$$
\left\|v_{\tau}\right\|^{\Omega} \leqslant O\left\|L_{\tau} v_{\tau}\right\|^{\Omega}, \quad \text { for each } v_{\tau} \in D_{L \tau} \text {, and each } \tau \in B \text {. }
$$


Now $u_{\tau}$ belongs to $D_{L \tau}$ by hypothesis and $\varphi^{2} w_{\tau}$ is also in $D_{L \tau}$ by prop. (3.5). Hence the inequality $(4.8)$ can be applied to $u_{\tau}-\varphi^{2} w_{\tau}$; and that gives

$$
\left\|u_{\tau}-\varphi^{2} w_{\tau}\right\|^{\Omega} \leqslant C\left\|\square_{\tau}\left(u_{\tau}-\varphi^{2} w_{\tau}\right)\right\|^{\Omega}, \quad \text { for } \operatorname{each} \tau \in B
$$

We recall that the choice of $w$ depends on $t$ and shall prove that

$$
\lim _{\tau \rightarrow i}\left\|\square_{\tau}\left(u_{\tau}-\varphi^{2} w_{\tau}\right)\right\|^{\Omega}=0 .
$$

Actually a direct application of the above inequalities $(4.3,4,6,7)$ gives

$$
\begin{aligned}
\left\|\square_{\tau}\left(u_{\tau}-\varphi^{2} w_{\tau}\right)\right\|^{\Omega} \leqslant \| \square_{\tau} u_{\tau}- & \square_{t} u_{t}\left\|^{\Omega}+\right\| \square_{t}\left(u_{t}-\varphi^{2} w_{\tau}\right) \|^{\Omega}+ \\
& +\left\|\left(\square_{\tau}-\square_{t}\right) \varphi^{2} w_{\tau}\right\|^{\Omega} \leqslant\left\|f_{\tau}-f_{t}\right\|^{\Omega}+k_{z_{2}} k_{3}|t-\tau|+k_{1} k|t-\tau| .
\end{aligned}
$$

The three terms of the last inequality are obtained as follows:

the first one from the fact that $\square u=f$, the second one from (4.4) and (4.6), the last one from (4.3) and (4.7). Since $f \in C_{q+1}(\bar{\Omega})$ the last inequality proves (4.10). Then the statement of prop. (4.5) can be derived from the fact that both terms at the left side of (4.6) and (4.9) tend to zero as $\tau$ tends to $t$. Q.E.D.

In order to prove the continuity of the solution $u$ we remark that, for every pair $A_{0}, A_{0}^{\prime}$, with $A_{0} \subset \subset A_{0}^{\prime} \subset \subset \Omega_{0}$ and $s \geqslant 0$, the constant appearing in the classical Friedrichs inequality

$$
\left\|v_{t}\right\|_{s+2}^{A} \leqslant \text { const }\left\{\left\|\square_{t} v_{t}\right\|_{s}^{A^{\prime}}+\left\|v_{t}\right\|^{A^{\prime}}\right\}, \quad \text { for each } v_{t} \in \tilde{C}_{q}\left(\bar{\Omega}_{t}\right),
$$

can be chosen indipendent of $t$. Actually, by the regularity of the coefficients $L_{A B \alpha}^{a b}(z, t)$ appearing in (4.2), the punctual ellipticity constant

$$
\left.c(z, t)=\min \left\{\sum_{a A}\left|\sum_{b B} \sum_{|\alpha|=2} L_{A B \alpha}^{a b}(z, t) \xi^{\alpha} \omega_{b}^{B}\right|, \xi \in \mathbb{C}^{n},|\xi|=1 ; \omega \in \mathbb{C}^{(n)}\right)^{n},|\omega|=1\right\} .
$$

is bounded from above by a positive constant on each coordinate set $W_{1}, \ldots, W_{N}$. Thus the standard Friedrichs procedure gives (4.11). First of all we deduce that, for each $A_{0} \subset \subset \Omega_{0}$ the norms $\left\|u_{\tau}\right\|_{3}^{A}$ are bounded indipendently from $\tau$. Actually by (4.11) we have

$$
\left\|u_{\tau}\right\|_{8+2}^{A} \leqslant \text { const }\left\{\left\|f_{\tau}\right\|_{8}^{\Omega}+\left\|u_{\tau}\right\|^{\Omega}\right\} \leqslant \text { const }\left\|f_{\tau}\right\|_{s}^{\Omega}
$$

the last inequality being derived by (4.8). Thus we ean write

$$
\left\|u_{\tau}\right\|_{s}^{A}<c, \quad \text { for each } s \geqslant 0
$$

where the constant $e$ depends only on $s$ and $A_{0}$. 
Proof of proposition (4.1). - Let $(z, t)$ and $(\zeta, \tau)$ be points in the same coordinate neighborhood $W_{j}$. We shall drop the subscript $j$ (which is fixed) because our algorithm is already sufficiently ornate. A straightforward application of the Sobolev inequality gives, for $s$ and $s^{\prime}$ large enough, $b \leqslant n,|B|=q$

$$
\left|u_{B}^{b}(z, t)-u_{B}^{b}(\zeta, \tau)\right| \leqslant \mathrm{const}\left\|u_{t}\right\|_{s^{\prime}}^{W}|z-\zeta|+\text { const }\left\|u_{t}-u_{\tau}\right\|_{s+2}^{W} .
$$

As, by (4.12), the first part tends to zero for $(\zeta, \tau) \rightarrow(z, t)$, hence we have only to prove that

$$
\lim _{\tau \rightarrow t}\left\|u_{t}-u_{\tau}\right\|_{s+2}^{W}=0
$$

Take some open subset $A_{0}$ of $X_{0}$ with $W \cap X_{0} \subset \subset A_{0} \subset \subset \Omega_{0}$ and apply (4.11) with $A$ replaced by $W$ and $A^{t}$ by $A$; that gives

$$
\left\|u_{t}-u_{\tau}\right\|_{s+2}^{W} \leqslant \text { const }\left\{\left\|f_{t}-f_{\tau}\right\|_{s}^{A}+\left\|\left(\square_{t}-\square_{\tau}\right) u_{\tau}\right\|_{s}^{A}+\left\|u_{t}-u_{\tau}\right\|^{A}\right\} .
$$

We now show that the three terms of the right hand side of the last inequality tend to zero as $\tau \rightarrow i$, this will prove (4.14) and the proposition.

The first term tends to zero because $f$ belongs to $O_{q}(\bar{\Omega})$; by $(4.3)$ the second one is smaller than $k_{1}|| u_{\tau} \|_{s+2}^{A}|t-\tau| \leqslant k_{1} e|t-\tau|$, see (4.12); the third one is smaller than $\left\|u_{t}-u_{\tau}\right\|^{\Omega}$ which tends to zero by prop. (4.5). Q.E.D.

(4.16) REMARE. - The inequality (4.13) is still valid if $D^{\alpha} u_{B}^{b}$ (derivatives only with respect to $z$ and $\bar{z}$ variables) replaces $u_{B}^{b}$, provided $s$ and $s^{\prime}$ are sufficiently large. This means that all derivatives of $u$ with respect to the variables $z$ and $\bar{z}$ are also continuous functions of $(z, i)$.

An immediate consequence of the above remark and of (3.8) is the following.

(4.17) Proposition. - If $\vec{\partial} f=0$ and $f_{t} \in R L_{t}, \forall t$ (the last condition is empty when $\Omega$ is Stein), then the form $v_{t}=\vartheta_{t} u_{t}$ is continuous in $\bar{\Omega}$ together with all its $z$ and $\bar{z}$ derivatives. It is the unique solution of the equation $\bar{\partial} v=f$ such that, for each $t \in B, v_{t}$ is orthogonal to the kernel of $T_{t}$.

For further applications we note that, for a fixed coordinate neighborhood $W \subset \subset \Omega$ we have

$$
\left|v_{t}\right|_{1}^{W} \leqslant \text { const }\left|f_{t}\right|_{n+1}^{\Omega},
$$

the constant depends only on $\Omega_{0}$ and $W_{0}$.

That follows from the Sobolev inequality $\left|v_{t}\right|_{1}^{W} \leqslant$ const $\left|u_{i}\right|_{2}^{W} \leqslant$ const $\|u\|_{n+3}^{W}$, the Friedrichs inequality (4.11) $\left\|u_{i}\right\|_{n+3}^{W} \leqslant$ const $\left\{\left\|f_{t}\right\|_{n+1}^{\Omega}+\left\|u_{t}\right\|_{0}^{\Omega}\right\}$, from (4.8), which gives $\left\|u_{t}\right\|_{0}^{\Omega} \leqslant C\left\|f_{t}\right\|_{0}^{\Omega}$, and the fact that $\left\|f_{t}\right\|_{n+1}^{\Omega}$ is smaller than const $\left|f_{t}\right|_{n+1}^{\Omega}$. 


\section{\$5. - Some lemmata for the product case.}

The following lemmas give some statement for the parametrized O.R. equation

$$
\bar{\partial} u=f
$$

for $C^{k}$ forms $(k=0, \ldots, \infty)$ of type $(0, q)$ with values in a $C^{k}$ family of holomorphic factor bundles on a single strongly pseudoconvex manifold $Y$ (there exists on $Y$ an exhaustive function which is plurisubharmonic outside a compact subset). The space of the parameter $t$ is the open ball $B$ of $\mathbb{R}^{m}$. The family mentioned above is a $C^{k}$ complex vector bundle $F \stackrel{p}{\rightarrow} Y \times B$ whose restrictions $F_{t} \stackrel{\text { def }}{=} F \mid(Y \times\{t\})$ are holomorphic bundles. Let $A_{0}^{0 \alpha}$ be the fiber bundle of $(0, q)$-forms on $Y$ and let $A^{00}$ be the pull-back of $A_{0}^{0 \alpha}$ by natural projection $Y \times B \rightarrow Y$. Our forms are sections of $\Lambda^{0 a} \otimes F$. The space of $C^{k}$ forms of this type whose restrictions to $Y \times\{t\}$ are $C^{\infty}$ sections of $F_{t}$ will be noted by $C_{\alpha}^{k}$. We note first that the problem (5.1) can be easily reduced to the equation

$$
\bar{\partial} u_{t}=f_{t}, \quad t \in B
$$

where $u_{t}$ and $f_{t}$ are $C^{k}$ families of sections of a single bundle $F_{0} \rightarrow Y$.

Actually, by well known results of Grauert on analytic bundles on Stein spaces ([2], see also [1]), since the bundles $F_{t}$ are continuously homotopic, they are isomorphic as holomorphic bundles and the isomorphism $\phi_{t}: F_{0} \rightarrow F_{t}$ can be chosen to be a $C^{\text {th }}$ homotopy. More precisely there exists a $C^{k}$ vector bundle isomorphism $\phi: F_{0} \times B \rightarrow F$ such that $\phi\left(F_{0} \times\{t\}\right) \subset F_{t}$ and $\phi_{t}: F_{0} \times\{t\} \rightarrow F_{t}$ is a holomorphic vector bundle isomorphism. For sections $u, f, \ldots$ etc. of $\Lambda^{0 a} \otimes F$ we shall note by $\tilde{u}, \tilde{f}, \ldots$ the $\phi^{-1}$-corresponding sections of $A^{0 \alpha} \otimes\left(F_{0} \times B\right)$. We have $\bar{\partial} \tilde{u}=(\bar{\partial} u)^{\sim} . O_{\alpha}^{k}$ has a natural Fréchet topology: Take a locally finite, relatively compact atlas $\left(U_{j}, z_{j}\right)$ of $Y$ such that $F_{0} \mid U_{j}$ is trivial for each $j$ and a basis $\varepsilon_{a}^{(j)}$ of $C^{\infty}$ sections of $F_{0} \mid U_{j}(a=1, \ldots$, $N=r k F)$. The $\varepsilon_{a}^{(j)}$ can be trivially extended as section $\tilde{e}_{a}^{(j)}$ of $\left(F_{0} \times B\right) \mid\left(U_{j} \times B\right)$ and $e_{a}^{(j)}=\phi \circ e_{a}^{(j)}$ are a basis of $O_{a}^{k}\left(U_{j} \times B\right)$ (in particular $F \mid\left(U_{j} \times B\right)$ is trivial). Any form $u \in O_{q}^{k}$ can be written as follows

$$
u(z, t)=\sum_{1}^{N} a_{j} \sum_{\left|A_{j}\right|=q} u_{A_{j}}^{a_{j}}(z, t) d \bar{z}_{j}^{A_{j}} \otimes e_{a}^{(j)}
$$

Then the Fréchet topology is induced by the seminorms

$$
|u|_{s}^{L}=\sup \left\{\left|D_{t}^{\alpha} D_{z_{j}}^{\beta} u_{a_{j}}^{A_{j}}\right|_{\left(z_{j}, t\right.},(z, t) \in L,\left(U_{j} \times B\right) \cap L \neq \emptyset, a_{j} \leqslant N,\left|A_{j}\right|=q,|\alpha| \leqslant k,|\beta| \leqslant s\right\}
$$

where $s$ is a non negative integer and $L$ is a compact subset of $Y \times B$. We note by $C_{q}\left(Y, F_{0}\right)$ the Fréchet space of $F_{0}$-valued $(0, q)$-forms of class $C^{\infty}$ on $Y . \phi$ induces a topological isomorphism between $C_{q}\left(Y, F_{0}\right) \hat{\otimes} C^{k}(B)$ and $C_{q}^{k}$ which commutes with 
the $\bar{\partial}$ operator. No confusion is possible for the topological tensor product because of the nuclearity of $C_{q}\left(Y, F_{0}\right)$. The first lemma we shall state occurs often in the literature and, roughly speaking, says that for a given differential operator $P: C^{\infty} \rightarrow C^{\infty}$, with closed range, the equation $P u_{t}=f_{t} \in R P$ admits a parametrized solution with the same regularity of $f_{t}$. A proof based on Künnneth formula for sheaves can be found in [9] prop. 10.1. We shall give here an other proof suggested by two Grothendieck's remarks.

Lemma. - For every colleetion $\left(b_{t}\right)_{t \in B}$ of forms $b_{t} \in C_{\alpha}\left(\bar{Y}, F_{0}\right)$ such that $\bar{\partial} b_{t}$ defines a form $g$ in $C_{a+1}^{k}$, there exists $v \in C_{q}^{k}$ such that $\bar{\partial} v_{t}=\bar{\partial} b_{t} ; k=0, \ldots, \infty$.

Proof. - Since the image $B_{q+1}=\bar{\partial} C_{\alpha}$ has finite codimension in $Z_{q+1}=$ ker $\left\{\bar{\partial}: C_{a+1} \rightarrow C_{a+2}\right\}$ which is closed, $B_{q+1}$ is also closed in the $C^{\infty}$ topology. By the Banach open map theorem, $\bar{\partial}$ induces a topological isomorphism $\bar{\partial}_{*}: O_{q} \mid Z_{q} \rightarrow B_{q+1}$. The $C^{k} \operatorname{map} \bar{\partial}_{*}^{-1} \circ \tilde{g}: B \rightarrow C_{q} / Z_{q}$ can be lifted to a $C^{k} \operatorname{map} \tilde{v}: B \rightarrow C_{q}$. Actually, for $k=0$ this is a lemma of Cartan $\left([1]\right.$, p. 119). For $0 \leqslant k<+\infty C^{k}(B)$ is a direct factor of a space of continuous functions on a locally compact space ([3] foot note p. 84) and so we are reduced to the case $k=0$. Finally, for $k=\infty$ the statement follows directly by the nuclearity of $C^{\infty}(B)$, see [3], pp. 83-84. Hence if we note by $p^{\prime}$ the natural projection $O_{q} \rightarrow O_{q} / Z_{q}$, we have $\bar{\partial} \tilde{v}=\bar{\partial}_{*} p^{\prime} \tilde{v}=\tilde{g}$, then $\bar{\partial} v=g=\bar{\partial} b$. Q.E.D.

In what follows we shall note by $Z_{a}^{k}$ the kernel of $\bar{\partial}: C_{a}^{k} \rightarrow C_{q+1}^{k}$.

$$
\text { LEMMa. - For } h \leqslant k, C_{q}^{k} \text { is dense in } C_{q}^{h} \text { and } Z_{q}^{k} \text { is dense in } Z_{\alpha}^{h} \text {. }
$$

Proof. - Since $C_{q}\left(Y, F_{0}\right)$ and $Z_{q}\left(Y, F_{0}\right)$ are nuclear, by th. 5 of [3], $C_{q}\left(Y, F_{0}\right) \hat{\otimes} C^{k}(B)$ is dense in $C_{q}\left(Y, F_{0}\right) \hat{\otimes} C^{h}(B)$ and $Z_{q}\left(Y, F_{0}\right) \hat{\otimes} C^{h}(B)$ is dense in $Z_{q}\left(Y, F_{0}\right) \hat{\otimes} C^{h}(B)$. Then the statement follows directly by applying the isomorphism $\phi$ to all these spaces. Q.E.D.

(5.5) Lemma. - For every fixed compact $L \subset Y \times B$, every $b \in C_{\alpha}^{k}$ such that $\bar{\partial} b \in C_{a+1}^{k+1}$ and $\varepsilon>0$, there exists $u \in C_{a}^{h+1}$ such that $\bar{\partial} u=\bar{\partial} b$ and $|b-u|_{k}^{L}<\varepsilon$.

Proof. - By lemma (5.3) there exists $v \in C_{a}^{k+1}$ such that $\bar{\partial} v=\bar{\partial} b$. Thus $v-b \in Z_{a}^{k}$. By lemma (5.4) there exists $w \in Z_{a}^{k+1}$ such that $|(b-v)-w|_{k}^{L}<\varepsilon$. Then $u=v+w$ is the solution. Q.E.D.

\$ 6. - a) In this first part of the present section we will refer implicitely to the special case that the vector bundle $E$ is the tangent bundle $T$ introduced in $\S 2$. A real vector field $w$ on $W$ is said to be Killing if it is projectable in the parameter space $B$ and its local one parameter group exp $\lambda w$ induces holomorphic diffeomorphisms between open subsets of the fibers $X_{t}$. In particular if $w$ is vertical (i.e. $\pi w=0$ ), then for fixed $\lambda \exp \lambda w$ is a local holomorphic automorphism on each 
fibre. Every vertical vector field $w$ can be uniquely written in the form $w=u+\bar{u}$, where $u=\sum_{\alpha} u_{\alpha}\left(\partial / \partial z_{\alpha}\right)$ is a section of $\boldsymbol{T}$. Then the condition for $w$ to be Killing is simply $\bar{\partial} u=0$. There are some particular vector fields on the patches $W_{j}$ defined in $\S 2 a$, they are $D_{i}^{v}=\left(\partial / \partial t^{\nu}\right)_{z_{j}=\text { const }}, \nu \leqslant m$; their local groups are the inverse images by $\varphi_{j}: W_{j} \rightarrow \mathbb{C}^{n} \times \mathbb{R}^{m}$ of the translations in that $t^{\nu}$-axis direction. The projection $\pi D_{j}^{v}$ is $\partial / \partial t^{v}$ and therefore, by the remark above, if we write $D_{j}^{v}-D_{i}^{v}=\theta_{j i}^{v}+\bar{\theta}_{j i}^{v}, \theta_{j i}^{v}$ becomes an holomorphic vector field on $X_{t} \cap W_{i} \cap W_{j}$ for fixed $t$. Clearly $\left(\theta_{j i}^{v}\right)_{j i}$ satisfies, by definition, the cocycle relation, thus, if one fix a partition of unity $p_{j}$ relative to the covering $\left(W_{j}\right)$, formula $g^{\nu} \mid W_{j}=\bar{\partial} \sum_{i} p_{i} \theta_{i j}^{v}$ defines a global $\bar{\partial}$-closed form $g^{v} \in C_{01}^{\infty}(w, T)$ which is not necessarely exact. $g^{v}$ is nothing else than the Dolbeault correspondent of the cocycle $\theta_{i j}^{v}$. In the classical theory of families of complex manifolds we have the following

(6.1) SCHOLIUM ([10]). - Let $U_{0}$ be a relatively compact open subset of $X_{0}$ and assume that the equations

$$
\bar{\partial} v^{v}=g^{v}, \quad v=1, \ldots, m
$$

admit a $C^{k}$ solution $(k=0, \ldots, \infty)$ in a neighborhood $\Delta$ of $U_{0}$ in $w$. Integration of the vector fields $\sum_{i} p_{i} D_{i}^{v}-v^{v}-\vec{v}^{v}$ gives, for $\lambda>0$ sufficiently small, a $C^{k+1}$ dif-. feomorphism $\tau: A_{0} \times B_{\lambda} \rightarrow W$ such that $\pi \circ \tau=\mathrm{pr}_{B}, \tau \circ \bar{\partial}=\bar{\partial} \circ \tau$, and $\tau(x, 0)=x$, $\forall x \in A_{0}:$

We shall also need a lemma of elementary analysis whose proof will be omitted:

(6.2) LeMMA. - Let $A^{\prime}, A^{\prime \prime}$ be open submanifolds of the riemannian manifold $A$, with $A^{\prime} \mathrm{CC} A^{\prime \prime C C} A$. Take vector fields $\xi^{\nu}(\nu \leqslant m)$ on $A \times B$ such that, for each $(a, t) \in A \times B, \xi^{p}(a, t)$ is tangent to $A \times\{t\}$, and suppose that $\xi^{\nu}(a, t)$ depends smoothly on $a$ and is of class $C^{k}$ as function of $(a, t)$. There exists a constant $\varrho$ such that, if $\left|\xi^{\nu}\right|<\varrho$, there is a unique diffeomeomorphism $\phi: A^{\prime \prime} \times B \rightarrow A \times B$ with the following properties

(i) $\operatorname{pr}_{B} \circ \phi=\operatorname{pr}_{B}$;

(ii) $\phi(a, 0)=a$, for each $a \in A^{\prime \prime}$;

(iii) $\phi\left(\partial / \partial t^{\nu}\right)=\xi^{\nu}+\partial / \partial t^{\nu}$;

(iv) $A^{\prime} \times B \subset \phi\left(A^{\prime \prime} \times B\right)$.

We shall denote by $C^{\infty k}$ the property of being $C^{k}$, and $C^{\infty}$ with respect to the first group of variables.

(6.3) Proposition. - Let $U_{0}$ be a relatively compact open subset of $X_{0}$ which admits a Stein neighborhood $\Delta_{0}$. There exists a neighborhood $\Omega$ of $U_{0}$ in $w$, two se- 
quences $\left(A_{N}\right)$ and $\left(A_{N}^{\prime}\right)$ of neighborhoods of $U_{0}$ in $X_{0}$ with

$$
A_{N}^{\prime} \subset \subset A_{N+1}^{\prime} \subset \subset A_{N+1} \subset \subset A_{N}
$$

and an injective $C^{\infty N+1}$ diffeomorphism $\tau_{N}: \bar{A}_{N} \times B \rightarrow$ w with the following properties

(i) $)_{N} \quad \operatorname{pr}_{B} \circ \tau_{N}=\pi$

(ii) $)_{N} \bar{\partial} \circ \tau_{N}=\tau_{N} \circ \bar{\partial}$ (i.e. $\tau_{N}$ sends $A_{N} \times\{t\}$ biholomorphically onto an open subset of $X_{i}$ );

(iii) $)_{N} \subset \tau_{N}\left(\bar{A}_{N}^{\prime} \times B\right)$;

(iv) $)_{N}$ The real vector field $\sum_{i} p_{i} D_{i}^{v}-\tau_{N}\left(\partial / \partial t^{v}\right)=v_{N}^{v}+\bar{v}_{N}^{y}$ (the equality defines $v_{N}^{v}$ ) is of class $O^{\infty N}$ and $v_{N}^{v}$ satisfies the equation

$$
\bar{\partial} v_{N}^{\nu}=g^{\nu} \quad \text { on } \tau_{N}\left(\bar{A}_{N} \times \bar{B}\right)
$$

(v) there is $\left|v_{N}-v_{N+1}\right|_{N}^{\bar{\Omega}}<2^{-N}$ on $\Omega$.

Proof. - Since $\Delta_{0}$ is Stein, $\Delta_{t}=\mathscr{C}^{-1}\left(\Delta_{0} \times\{t\}\right)$ is also Stein for $|t|$ small (see $\S 2 a$ ). Hence $L_{01}^{2}\left(\Delta_{t}, \boldsymbol{T}\right) \subset R L_{t}$, in particular $g_{t}^{v} \in R L_{t}$, thus, by prop. (4.17) there exists a $C^{\infty 0}$ solution $v_{0}$ of $(6.5)_{0}$ on $A$. By the above seholium there exist, an open neighborhood $A_{0}$ of $\bar{U}_{0}$ in $A_{0}$ and, for $B=\{|t|<\delta\}$ small enough an injective $C^{\infty 1}$ diffeomorphism $\tau_{0}: A_{0} \times B \rightarrow W$ which satisties (i $)_{0}$, (ii) $)_{0}$. Condition (iv) $)_{0}$ is also satisfied by construction of $\tau_{0}$. Let $A_{0}^{\prime} \subset \subset A_{0}$ be a neighborhood of $\bar{U}_{0}$ in $X_{0}$, we put $\Omega=\tau_{0}\left(A_{0}^{\prime} \times B\right)$, thus (iii) $\rangle_{0}$ is trivially satisfied. We proceed now by induction on $N$. Choose open sets $A_{N+1}, A_{N+1}^{\prime}$ of $X_{0}$ such that (6.4) is satisfied and let $\varrho_{N}$ be the smallest $\varrho$ given by lemma (6.2) applied to the situations $A_{N}^{\prime} \subset C A_{N+1}^{\prime} \subset C A_{N+1}$ and $A_{N+1}^{\prime} \subset C A_{N+1} \subset C A_{N}$. Since $v_{N}^{y}$ satisfies $(6.5)_{N}$ on $\tau_{N}\left(\bar{A}_{N} \times \bar{B}\right)$, by (ii) $)_{N}$ we have on $\bar{A}_{N} \times \bar{B} \bar{\partial}\left(\tau_{N}^{-1} v_{N}^{v}\right)=\tau_{N}^{-1} g^{v} \in C^{\infty N+1}\left(\bar{A}_{N} \times \bar{B}\right)$. Thus by lemma $(5.5)$ there exists $w^{\prime \prime} \in C^{\infty N+1}$. $\cdot\left(\bar{A}_{N} \times \bar{B}\right)$ such that $\bar{\partial} w^{v}=\tau_{N}^{-1} g^{v}$ and $w^{v}$ is arbitrarely close to $\tau_{N}^{-1} v_{N}^{v}$ in the $O^{N}$-norm. Thus we may assume that

$$
\begin{array}{ll}
\left|w^{v}-\tau_{N}^{-1} v_{N}^{v}\right|<\frac{1}{2} \cdot \varrho_{N} & \text { on } \bar{A}_{N} \times \bar{B}, \\
\left|\tau_{N} w w^{\nu}-v_{N}^{v}\right|_{N}<2^{-N} & \text { on } \tau_{N}\left(\bar{A}_{N} \times \bar{B}\right) .
\end{array}
$$

Put now $v_{N+1}^{v}=\tau_{N} w^{v}, u_{N+1}^{v}=\sum_{i} p_{i} D_{i}^{v}-v_{N+1}^{v}-\bar{v}_{N+1}^{\nu}$. Thus (v) follows immediately from (6.7) and (iii) $)_{N}$. We note that, although $\tau_{N}$ is not of class $C^{N+2}$, vector fields of class $C^{N+1}$ which are tangent to the fibers $A_{N} \times\{t\}$ are sent by $\tau_{N}$ into fields of the same class. The reason of this fact is that $\tau_{N}$ is of class $C^{\infty N+1}$ and that, by transporting such fields only a partial jacobian matrix of $\tau_{N}$ is involved, precisely the one where only derivatives with respect to the first group of variables appear and this matrix is of class $C^{N+1}$. This is the point where we gain one derivative. So $v_{N+1}^{v}$ and $u_{N+1}^{\nu}$ defined above are of class $C^{\operatorname{coN} N+1}$ on $\tau_{N}\left(\bar{A}_{N} \times \bar{B}\right)$. 
Now, from the identity $u_{N+1}^{v}-\tau_{N}\left(\partial / \partial t^{v}\right)=\left(v_{N}^{v}-v_{N+1}^{v}\right)+\left(\bar{v}_{N}^{v}-\bar{v}_{N+1}^{v}\right)$ on $\tau_{N}\left(\bar{A}_{N} \times \bar{B}\right)$ we get $\tau_{N}^{-1} u_{N+1}^{v}-\partial / \partial t^{v}=\left(\tau_{N}^{-1} v_{N}^{v}-w^{v}\right)+\left(\bar{\tau}_{N}^{-1} \bar{v}_{N}^{v}-\bar{w}^{\nu}\right)$ on $\bar{A}_{N} \times \bar{B}$. Hence by $(6.6)$ we have $\left|\tau_{N}^{-1} u_{N+1}^{\nu}-\partial / \partial t^{\nu}\right|<\varrho_{N}$ on $\bar{A}_{N} \times \bar{B}$. Let $\phi: \bar{A}_{N+1} \times \bar{B} \rightarrow A_{N} \times \bar{B}$ be the map defined in lemma (6.2) for the vectorfields $\tau_{N}^{-1} u_{N+1}^{v}-\partial / \partial t^{v}$ and the sets $A_{N+1}^{\prime}, A_{N+1}, A_{N}$.

We have

$$
A_{N}^{\prime} \times B \subset \phi\left(A_{N+1}^{\prime} B\right)
$$

Put $\tau_{N+1}=\tau_{N} \circ \phi$. We have $\tau_{N+1}\left(\partial / \partial t^{\nu}\right)=u_{N+1}^{v}$, hence $\tau_{N+1}$ is of class $C^{\infty N+2}$.

Conditions (i) $)_{N+1}$ and (ii) $)_{N+1}$ follow directly by the same properties verified by $\tau_{N}$ and $\phi$. Condition (iii) $)_{N+1}$ can be derived from (iii) $)_{N}$ by applying $\tau_{N}$ to both terms of (6.8). Finally the vector fields $v_{N+1}^{v}$ are $C^{\infty N+1}$ in $\tau_{N+1}\left(A_{N+1} \times B\right) \subset A_{N} \times B$ as we have already shown and we have $\bar{\partial} v_{N+1}^{v}=\tau_{N} \bar{\partial} w^{v}=g^{v}$, thus $(6.5)_{N+1}$ is also satisfied. Q.E.D.

Proof of Theorem (1.2). - As we have seen there exists a neighborhood $L$ of $K$ in $w$ which meets each $X_{t}$ in a Stein open set $L_{t}$. Hence there exists, for each $t, u_{t}^{\prime} \in C^{\infty}\left(L_{t}, E_{t}\right)$ such that $\bar{\partial}_{t} u_{t}^{\prime}=f_{t}$. We do not know how $u_{t}^{\prime}$ depends on $t$. Now, by (iv) and (v) of prop. (6.3) $v^{\prime \prime}=\lim _{N \rightarrow \infty} v_{N}$ is a solution of equation (6.5) in a neighborhood (in $W$ ) of $K$. Hence, by scholium (6.1) there exists $A_{0}$, with $K \subset A_{0} \subset X_{0}$, $\lambda>0$ and an injective diffeomorphism $\tau: A_{0} \times B_{\lambda} \rightarrow W$ such that $\pi \circ \tau=p_{B_{2}}$, $\tau \circ \vec{\partial}=\bar{\partial} \circ \tau$ and $\tau(x, 0)=x, \forall x \in A_{0}$. We can assume that $\Omega \stackrel{\text { def }}{=} \tau\left(A_{0} \times B_{\lambda}\right)$ is contained in $L$. Apply lemma (5.3) to the vector bundle $\tau^{-1} E \rightarrow A_{0} \times B$ and the collection of forms $\left(\tau^{-1} u_{t}^{\prime}\right)$. Since $\bar{\partial} \tau^{-1} u^{\prime}=\tau^{-1} f \in C_{0, \alpha+1}^{\infty}\left(A_{0} \times B_{\lambda}, \tau^{-1} E\right)$ there exists $v \in C_{0, q}^{\infty}\left(A_{0} \times B_{\lambda}, \tau^{-1} E\right)$ such that $\bar{\partial}_{t} v_{t}=\bar{\partial}_{t} \tau^{-1} u_{t}^{\prime}=\tau^{-1} f_{t}$. Hence $u=\left.\tau v\right|_{\Omega} \in C_{0 q}^{\infty}(\Omega, E)$ is the required solution of (1.1). Q.E.D.

\section{\$ 7. - Applications.}

a) Pseudorigidity of Stein Manifolds for $0^{\infty}$ deformations.

Definitron. - A complex manifold $X_{0}$ is said to be pseudorigid for $C^{\infty}$ deformations if, for every $C^{\infty}$ family of complex manifolds in which $X_{0}$ is the manifold at the time $t=0$ and every compact subset $K$ of $X_{0}$, there exist an open neighborhood $A_{0}$ of $K$ in $X_{0}$ and a map $\tau$ as in scholium (6.1).

Thus scholium (6.1) and theorem (1.2) say that every Stein manifold is pseudorigid for $C^{\infty}$ deformations.

\section{b) The complex Frobenius theorem.}

The last statement on deformations permits to give a very simple proof of the following theorem of L. Nizenberg ([8]).

12 - Annali di Matematica 
Theoreir (L. NIRENBerg). - Let $\omega_{1}, \ldots, \omega_{n}, \lambda_{1}, \ldots, \lambda_{p}$ be $0^{\infty}$ complex 1-forms on $\mathbb{R}^{N}$ indipendent at each point and let $\Omega(y)$ be the space generated over $\mathbb{C}$ by them at $y$. Suppose that the space $\Lambda=\Omega \cap \bar{\Omega}$ has constant dimension $p$. Then a (necessary and) sufficient condition for the existence of local coordinates $x_{1}, \ldots, x_{2 n}, t_{1}, \ldots, t_{p}, s_{1}, \ldots, s_{q}$ such that $\Omega$ is generated at each point by the differentials of $z_{j}=x_{i}+i x_{i+n}(j \leqslant n)$ and by $d t_{1}, \ldots, d t_{p}$ is that

$$
d \Omega \subset J \Omega, \quad d \Lambda \subset J \Lambda
$$

$\Omega$ stays for the space of $C^{\infty}$ forms which are in $\Omega$ at each point, $d \Omega$ stays for the space of differentials of forms in $\Omega$ and $J \Omega$ stays for the ideal generate by $\Omega$ in the exterior algebra of all $C^{\infty}$ complex forms.

We now give a proof which is based on Newlander-Nirenberg theorem (i.e. the above theorem in the case that $\Lambda=0$ and that $\Omega+\bar{\Omega}$ is the space of complex 1-forms on $\mathbb{R}^{N}$ ) and on pseudorigidity of Stein manifolds. Put $\Gamma=\Omega+\bar{\Omega}$. Since $2 \operatorname{dim} \Omega=\operatorname{dim} \Gamma+\operatorname{dim} \Lambda$, we have $\operatorname{dim} \Gamma=2 n+p$. Put $q=N-2 n-p$. Since $\Lambda=\bar{\Lambda}$ and $\Gamma=\bar{\Gamma}$, the spaces $\Lambda_{R}$ and $\Gamma_{R}$ of forms of $\Lambda$ and $\Gamma$ with real coefficients generate them as follows: $\Lambda=\Lambda_{R}+i \Lambda_{R}, \Gamma=\Gamma_{R}+i \Gamma_{R} . \Lambda$ can be supposed to have $\lambda_{1}, \ldots, \lambda_{p}$ as basis and $\lambda_{1}, \ldots, \lambda_{p}$ can be supposed to belong to $\lambda_{R}$. One can choose $\gamma_{1}, \ldots, \gamma_{2 n}$ in $\Gamma_{R}$ such that $\lambda_{1}, \ldots, \lambda_{n}, \gamma_{1}, \ldots, \gamma_{2 n}$ is a basis of $\Gamma_{n}$ over $\mathbb{R}$ and of $\Gamma$ over $\mathbb{C}$. Conditions (7.1) may be rewritten as follows

$$
d \omega_{j}=\sum_{1}^{n} h \chi_{j}^{h} \wedge \omega_{h}+\sum_{1}^{D} \theta_{j}^{m} \wedge \lambda_{m}, \quad j \leqslant n ; \quad d \lambda_{m}=\sum_{1}^{p} \varrho_{m}^{k} \wedge \lambda_{k}, \quad m \leqslant p
$$

where $\chi_{j}^{n}, \theta_{j}^{m}$ and $\varrho_{m}^{k}$ are suitable 1 -forms. Since the $\gamma$ 's are combinations of $\omega$ 's, (7.2) gives $d \gamma_{j}=\sum_{1}^{2 m} \mu_{j}^{h} \wedge \gamma_{j}+\sum_{i}^{p} \nu_{j}^{m} \wedge \lambda_{m}$. Addition to this identity and to $(7.2)_{2}$ of their respective conjugates shows that the forms $\varrho_{m}^{k}, \mu_{j}^{h}$ and $\nu_{j}^{m}$ may be assumed to have real coefficients, hence $d \Lambda_{R} \subset \mathfrak{J}_{R} \Lambda_{R}$ and $d \Gamma_{R} \subset \mathfrak{J}_{R} \Gamma_{R}$, where $\mathfrak{J}_{R}$ stays for ideal generated over real forms. Thus, by the classical Frobenius theorem there exist coordinates $y_{1}, \ldots, y_{2 n}, t_{1}, \ldots, t_{p}, s_{1}, \ldots, s_{q}$ such that $A_{R}$ is generated by $d t_{1}, \ldots, d t_{p}$, say $\Lambda_{R}=\mathbb{R} d t$, and $\Gamma_{R}=\mathbb{R} d y \oplus \mathbb{R} d t$, hence $A=\mathbb{C} d t, \Gamma=\mathbb{C} d y \oplus \mathbb{C} d t$ and $\Omega=\mathbf{C} \omega \oplus \mathbb{C} d t$. The terms of the forms $\omega_{j}(j \leqslant n)$ which contain the differentials $d t_{1}, \ldots, d t_{p}$ may obviously be suppressed and since all forms of $\Gamma$ do not contain the differentials $d s_{1}, \ldots, d s_{q}$, we can write $\omega_{j}=\omega_{j}(y, t, s)=\sum_{1}^{n} \omega_{j n}(y, t, s) d y^{h}$. Fix now $t, s$ and take on the ball $B$ of ray $\delta$ of $\mathbb{R}^{2 n}$ the distribution $\Omega_{t_{s}}(y)=\mathbb{C} \omega(y, t, s)$. Since the jacobian $\left(\omega_{j k}, \bar{\omega}_{i k}\right)$ is non degenerate, all complex 1 -form of $\mathbb{R}^{2 n}$ are generated by $\Omega_{t s}, \bar{\Omega}_{t s}$ and $\Omega_{t s} \cap \bar{\Omega}_{t_{s}}=\{0\}$. Thus we can apply Newlander-Nirenberg theorem and get a complex structure $B_{t_{s}}$ on $B$. Since the function $|y|^{2}$ is plurisubharmonic near the origin of $B_{t_{s}}$, for $\delta$ small enough $B_{00}$ is Stein. We apply now the pseudorigidity theorem (a), thus by successive shrinking of $B_{00}$ if needed, we have a neighborhood $U$ 
of the origin of $\mathbb{R}^{N}$ and a biholomorphic map $\phi_{t s}: B_{t s} \cap U \rightarrow B_{00}$ which depends smoothly on $(y, t, s)$ : thus $\phi_{t_{s *}} \Omega_{00}=\Omega_{t_{s}}$. Finally take complex coordinates $\xi_{i}=\xi_{j}+i \xi_{j+n}(j \leqslant n)$ on $B_{00}$ and put $x_{j}(y, t, s)=\xi_{j}\left[\phi_{t s}(y)\right],(j \leqslant 2 n)$. We claim that $x, t, s$ are the desired coordinates. Actually $d z=\phi_{t s *} d \zeta+\sum_{1}^{p} a_{h} d t^{h}$. Since $\phi_{t s *} d \zeta \in \Omega_{t_{s}}$ we have $d z \in \mathbb{C} \omega+\mathbb{C} d t=\Omega$. The rest follows by straightforward dimensional arguments. Q.E.D.

c) A direct proof can be given by putting on $B \subset \mathbb{R}^{2 n}$ the almost complex structure $\Omega_{t s c}(y)=\mathrm{C} \omega(c y, t, s)$ and proceeding as in the classical proof: assume that $\omega_{j}(0,0,0)=d y_{j}+i d y_{j+n}$ and apply (4.18) with $f_{t}$ replaced by the $\bar{\Omega}_{t s c}$-component of $d y_{j}+i d y_{j+n}$. For fixed $c$ small enough, prop. (4.17) and theorem (1.2) give complex functions $v_{t s c}^{j}, j \leqslant n$, such that the $n$ functions $z_{j}(y, t, s)=\left(y_{j}+i y_{j+n}\right) / c-$ $-v_{t s c}^{j}(y / c), j \leqslant n$, together with $t$ and $s$ give the desired coordinates.

\section{BIBLIOGRAPHY}

[1] H. CARTaN, Espaces fibrés analytiques, Symposium Internacional de Topologia algebraica, Mexico, 1958.

[2] H. GRAUERT, Analytische Faserungen ueber holomorph-vollstaendigen komplexen Raeumen, Math. Ann., 135 (1958), pp. 263-273.

[3] A. GRothendieck, Résumé des résultats essentiels dans la théorie des produits tensoriels topologiques et des espaces nucléaires, Ann. Inst. Fourier, 4 (1952), pp. 73-112.

[4] L. HöRMANDER, $L^{2}$-Estimates and existence theorems for the $\bar{\partial}$ operator, Acta Math., 113 (1965), pp. 89-152.

[5] L. HöRMANDER, Introduction to analysis in several complex variables, D. Van Nostrand (1966).

[6] K. KODAIRA - D. C. SPENCER, On deformations for complex analytic structures, I, II, Ann. of Math., 67 (1958), pp. 328-466.

[7] J. J. KoHN, Harmonic integrals on strongly pseudoconvex manifolds, I, II, Ann. of Math., I, 78 (1963), pp. 112-148, II, 79 (1964), 450-472.

[8] L. Nirenberg, $A$ complex Frobenius theorem, Conf. on analytic functions, Inst. for adv. Study, 1958.

[9] C. REA, Le problème de Cauchy-Riemann pour des structures mixtes, Ann. Se. Norm. Sup. Pisa, 22 (1968), pp. 695-727.

[10] C. REa, Deformazioni di strutture su varietà, Rend. Sem. Mat. Torino, 30 (1970-71), pp. $149-164$. 